\title{
Current perspectives in the treatment of small cell lung cancer
}

\author{
Andrew T. Turrisi, III \\ Radiation Oncology, University of Michigan Medical Center, Ann Arbor, Michigan, USA
}

\section{Summary}

Neither chemotherapy nor radiotherapy alone is adequate to treat both bulky local disease and the almost universal distant micrometastases of small cell lung cancer (SCLC). Despite improved overall and 2-year survival rates associated with combining the two treatment modalities, however, their potential for toxic interaction demands careful consideration. The specific toxicity profile of the chemotherapeutic agent used must be calculated and balanced with the radiotherapy dose, fractionation, volume, and timing with chemotherapy to give the patient the maximum benefit and the least amount of risk. Results of clinical trials indicate that fractionation of the radiation dose takes advantage of the fact that fractionation causes less damage to and allows for repair of normal tissue, whereas the tumor cells of SCLC are killed exponentially by even small radiation doses per fraction. Further evaluation of radiation volume is needed to answer questions on the risk-benefit ratio of normal lung exposure versus complete coverage of areas of potential metastasis, and to determine whether dose of volume is the more critical factor for lung toxicity. Finally, the timing of radiotherapy also must be studied further. Early radiotherapy offers the potential for killing small cells before they migrate, but attempts to compensate for such early exposure may lead to subtherapeutic doses of chemotherapy and, thus, to lower response rates.

Key words: Combined-modality therapy; Small cell; Thoracic radiotherapy; Prophylactic cranial irradiation

\section{Introduction}

Since the 1960s, progress in the treatment of small cell lung cancer (SCLC) has been encouraging. Admittedly, some of this progress may be the result of improved staging

Correspondence to: Andrew T. Turrisi, III, UH B2C490, Box 0010, 1500 E Medical Center Drive, Ann Arbor, MI 48109, USA. 
techniques and the inclusion of only those patients with limited-stage disease in clinical trials, but significant changes in our approach to the management of SCLC have also affected outcome favorably.

In the 1960s it became apparent that surgery alone did not completely resect SCLC, as disease in these patients rapidly progressed with distant metastases. Radiotherapy provided better local control and survival for most patients, but also proved incapable of averting disease dissemination. Subsequently, chemotherapy became the cornerstone of therapy for SCLC, whether limited or extensive.

The need for chemotherapy is unquestioned, since the great majority of patients with SCLC have a form of extensive disease. Even when SCLC is categorized as limited to the chest, only rarely are distant micrometastases absent. Nevertheless, in limited disease the metastases are clinically occult-beneath the level of detection of current imaging modalities, which require at least $10^{8}$ cells to be visualized.

Despite improved response rates with chemotherapy during the 1970s and early 1980s, survival rates were only marginally affected; median survival rarely exceeded 12-14 months. By itself, then, chemotherapy was unable to manage bulky disease of the chest adequately, and, ultimately, intrathoracic recurrence was seen in $80-100 \%$ of patients so treated. For this reason, radiotherapy was reintroduced to the therapeutic regimen. However, early attempts to use chemoradiotherapy were marred by excess to toxicity.

\section{Combination Chemotherapy/Radiotherapy}

A 1983 review of the literature by the International Association for the Study of Lung Cancer [1] noted that chemotherapy in combined-modality trials was generally based on cyclophosphamide, most commonly combined with doxorubicin and vincristine. These trials, which added continuous or split-course radiotherapy to cyclophosphamide-based chemotherapy showed improved survival in only two of the seven trials. The methods of combining chemotherapy with radiotherapy, the cyclophosphamide-based chemotherapy, split-course, low-dose, early technology may explain these results. However, because of the diagnostic imaging revolution, it is difficult and imprecise to compare studies from more than a decade ago with results today.

\section{Dangerous liaisons: toxicities of combination therapy}

Combining thoracic irradiation with doxorubicin (a cardiotoxin), bleomycin, mitomycin, or the nitrosoureas (lung toxins), greatly increases the risk of cardiac, esophageal, and lung toxicity associated with radiotherapy alone. Usual subliminal or rare toxicities may be promoted to more commonplace events. The expected timing of toxicities may also be altered. Radiation pneumonitis usually occurs at 2-6 months. When bleomycin, lomustine, or mitomycin is used, pneumonitis can be seen much earlier, even during radiotherapy. Obviously, the damage is caused by the therapy, but its manifestation is delayed until months later. Using both modalities eliminates this latency period. This suggests that the chemotherapeutics may lower the threshold for radiation pneumonitis, perhaps in these instances better called combined-modality pneumonitis. 


\section{Considerations for planning radiotherapy}

Radiation technology has evolved significantly over the past 90 years. Magnetic resonance and computed tomography (CT) imaging techniques, for example, have improved to allow more specific disease localization and radiation targeting, while simulators and CT scanners provide more accurate planning of radiotherapy. Radiotherapy planning involves steps to design radiotherapy field sizes as well as sizes, shapings, angles, and depths of the fields. These procedures allow a tumor coverage to be optimized, while limiting the exposure of normal tissues. Dose, fractionation, volume, and timing with chemotherapy are the four important considerations concerning the influences of radiotherapy in lung cancer.

\section{Dose and fractionation}

The total radiation dose and fractionation - or the daily dose and total time to achieve that dose - influence not only the actual physical dose but also its effective biologic impact. Altered fractionation and combination with chemotherapy change acute effects to tumor and normal tissues and late effects, such as local control of tumor and normal tissue fibrosis in lung, strictures to the oesophagus, and genetic damage leading to second tumors. Commonly, in former methods, chemotherapy was administered first, followed by radiotherapy (the sequential method). Retrospective analysis of the effect of dose on locoregional failure rates shows that, at lower doses, disease recurs in $80-90 \%$ of patients within a 3-year period (Fig. 1). Even at doses of 40, 45 and $50 \mathrm{~Gy}, 30-50 \%$ of patients will experience local recurrence [2]. Clearly, these doses administered sequentially are inadequate for local control; when chemotherapy alone is used, local failure occurs in $80-100 \%$ of patients [3].

Underlying a potential importance of fractionation are tissue culture observations

\section{LIMITED-STAGE SCLC}

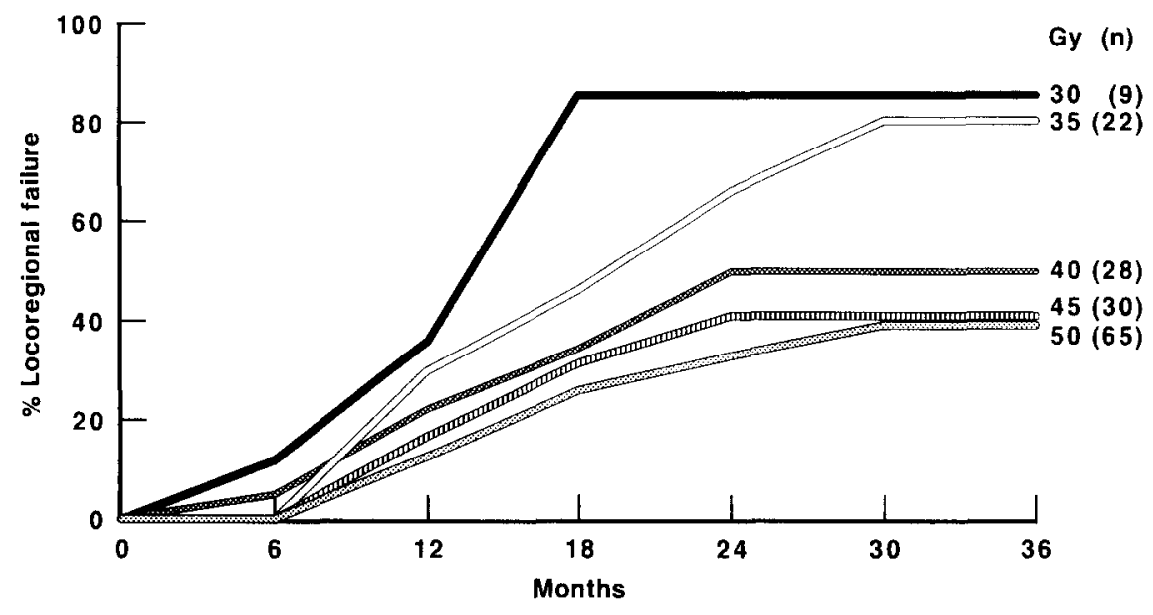

Fig. 1. Effect of radiation dose on locoregional failure rate. 
about radiation response in normal tissues and tumors. Most normal tissues and tumors have an initial region where dose appears to accumulate before exponential cell killing is observed. This region is called the radiobiologic shoulder. Classic SCLC cells have no shoulder; lymphocytes are a normal cell population with this characteristic. Thus, smaller fractions of radiation kill SCLC exponentially at doses that merely damage many normal tissues [4]. Exploiting this difference provides a potentially therapeutic advantage. Small fractions cause less damage to tissues with a radiobiologic shoulder, like normal lung and spinal cord tissue. At the same time, the interval between fractions allows both repair of sublethal damage to normal tissue and passage of the surviving SCLC cells from the relatively resistant $S$-phase of the cell cycle to the more sensitive $G_{2-}$ or $M$-phase. In view of the very short doubling time and rapid growth of SCLC, cycle redistribution may be important. Unfortunately, the non-SCLC histologies and the variant SCLCs have radiobiologic shoulders, so there may be less reason for hyperfractionation schemes. Nevertheless, the prospect of repopulation of tumor during protracted fractionation schedules continues to make accelerated schemes an appealing strategy. Thus, there is a special reason to attempt altered fraction schemes in limited SCLC.

\section{Volume}

The radiation field must balance the necessary exposure of tumor cells with the need to protect normal tissue. Entering and exiting beams expose normal tissues. Standard techniques include a margin of normal tissue to allow for a variation in setup, tumor motion, uncertainties of tumor extent, and physical buildup of dose. Older radiotherapy techniques applied to protect the spinal cord commonly block radiation to the mediastinum, which, prior to CT scans, was a poorly visualized area that commonly harbored bulky subcarinal nodes. At the same time, clinically uninvolved contiguous areas of lymphatics have been irradiated. This expands the volume of normal tissue irradiated. As the Halsted or 'en bloc' approach was initially applied to breast cancer, these expanded volumes have been attempted in lung cancers. Similarly, the success of expanded fields in the treatment of Hodgkin's disease unfortunately has not been mirrored when these strategies have been applied to lung cancer. Since contiguous spread to lymphatics seems isolated to Hodgkin's disease and the 'en bloc' approach has faltered elsewhere, it should not be surprising that treatment of neighboring nodal regions does not improve outcome in lung cancer. Supraclavicular nodes are infrequent sites of exclusive failure, and failure at these sites has been associated with distant rather than local failure [5]. Clearly, expansive radiotherapy fields expose larger areas of normal tissue to toxicity and, while such a compromise might be acceptable were radiotherapy used alone, volume merits careful reconsideration when combined modalities are used.

When the target volume is limited to the tumor, first echelon nodes, and less generous margins of normal tissues, methods can be designed to treat this target and expose the spinal cord and lung to lower, more tolerable doses. Many propose that the prechemotherapy tumor volume dictates the target. This clearly is the case for concurrent chemoradiotherapy during Cycle 1 treatment since there is no reduced volume. However, one advantage of delaying thoracic therapy to later cycles is the potential to trim the volume. Whether this reduces local control or compromises survival has not been subjected to trial. When patients achieving a complete response were randomized to radiotherapy versus observation, a Southwest Oncology Group (SWOG) trial could demonstrate no benefit to 
the radiotherapy [6] but analyzing volume in this study suggested that the volume irradiated had little influence on either survival or local control.

Two trials have looked at the timing-early versus delayed-of thoracic radiotherapy. In one such study, delaying thoracic radiotherapy to Cycle 4 allowed full doses of chemotherapy to be given and, compared with chemotherapy alone or with immediate radiotherapy at Cycle 1, produced the best survival [7]. Murray and colleagues [8] in a National Cancer Institute of Canada trial randomized patients to platinum/etoposide with concurrent radiotherapy at either Cycle 2 or Cycle 6 and observed a survival benefit for early radiotherapy.

\section{Clinical Application of Radiothepapy/Chemotherapy}

Three methods of integrating thoracic radiotherapy and chemotherapy have been used in limited SCLC trials: sequential, alternating, and concurrent. The sequential strategy appears to have inferior results. This may be due to types of chemotherapy used or suboptimal doses, volumes, or fractionation schemes. Studies comparing alternating or concurrent methods to sequential methods need to use programs providing equal toxicitywhich may be difficult to estimate.

The alternating approach to integrating chemotherapy and radiotherapy uses both treatments at full dose whenever possible, with radiation administered in split courses. Usually, the therapies are alternated rapidly, with onc modality used one weck and the other used the following week. In the third week, treatment returns to that of week 1 or is delayed to allow recovery from the toxic effect of the first 2 weeks.

Numerous clinical trials have incorporated technically different radiotherapeutic approaches with chemotherapy. A reanalysis of the 1970 National Cancer Institute (NCI) data suggested that 3 weeks of concurrent chemoradiotherapy was optimal [9].

Coupled with the biologic information that SCLC lacks a shoulder, these data formed the underpinning of a trial initiated at the University of Pennsylvania in 1984 [5].

The induction schema of the Penn Study is shown in Fig. 2. Cisplatin $60 \mathrm{mg} / \mathrm{m}^{2}$ was given on day 1 and etoposide $120 \mathrm{mg} / \mathrm{m}^{2}$ on days 4,6 and 8 . Thoracic radiotherapy was administered twice daily and concurrently in this study, beginning on day 1 . On day 22 , patients were given full Cycle 2 doses of cisplatin/etoposide, irrespective of nadir blood counts or severity of esophagitis. All radiotherapy was completed by day 21 , delivering a tumor dose of $45 \mathrm{~Gy}$ in 3 weeks.

Of 32 patients in this trial, $73 \%$ developed Grade 3 or 4 myelotoxicity. In fact, data from subsequent Eastern Cooperative Oncology Group (ECOG) and other groups support that Grade 3 or 4 leukopenia appears to develop in $60-75 \%$ of patients treated with comparable regimens [10-12]. Acute esophageal toxicity (Grade 2, mild difficulty in swallowing, or Grade 3, able to swallow liquids but not solids) were each noted in approximately $40 \%$ of patients in the original Penn and the subsequent ECOG confirmatory trial, which was a multi-institutional pilot study of the same treatment program. In contrast to earlier trials that used cyclophosphamide-based chemotherapy, however, esophageal toxicity was reversible in these patients; no strictures were produced in long-term survivors [10].

Survival data for the concurrent cisplatin/etoposide/twice-daily thoracic radiotherapy studies show that disease-free survival remains at approximately $50 \%$, but the different in- 
Induction Schema

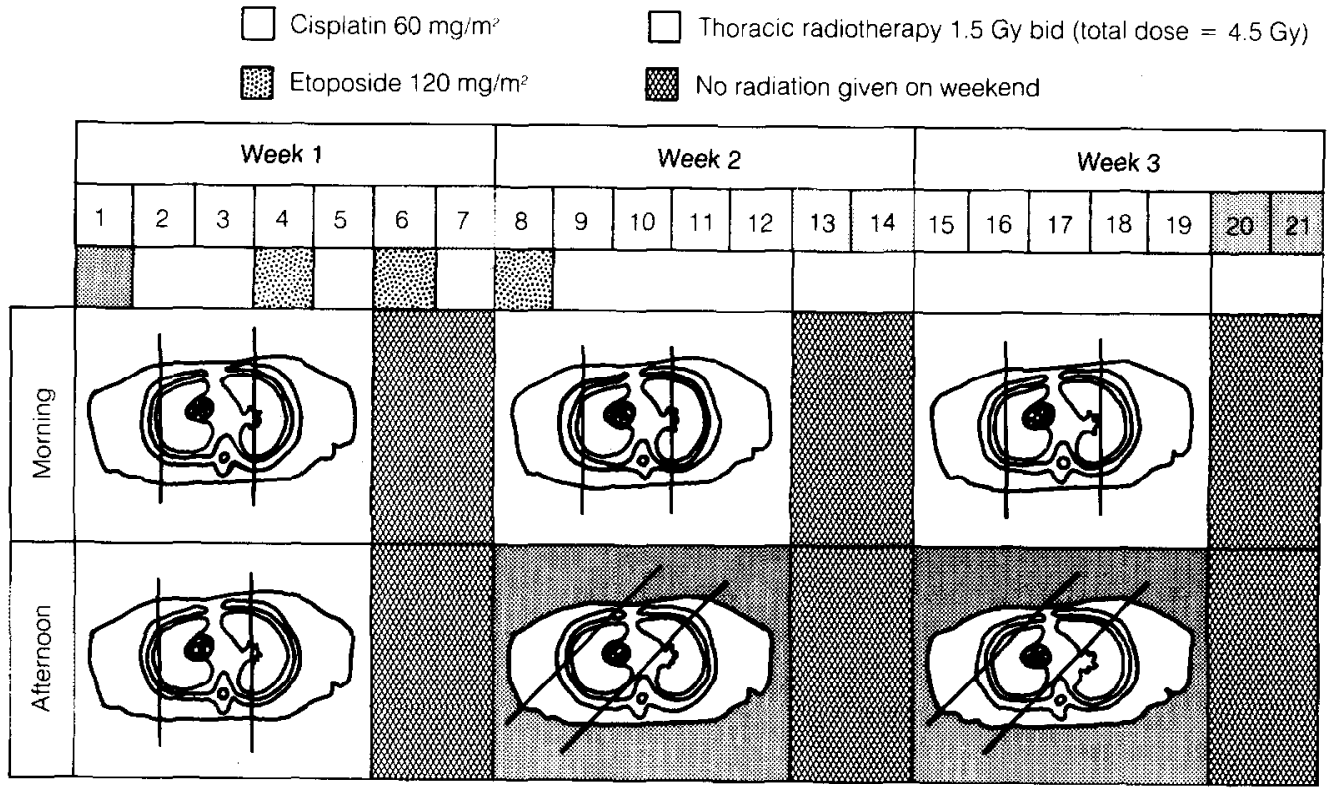

Fig. 2. Induction schema for trial of cisplatin/etoposide and thoracic radiotherapy. Radiation fields are shown. Reprinted from Turrisi et al. (see Ref. 5) with permission.

stitutions have discordant disease relapse patterns beyond 2 years. At a minimum followup of 48 months, 5-year disease-free survival is still flat at $40-50 \%$ in the Penn study [17] (Fig. 3).

Comparing these figures globally with studies by SWOG [13], NCI [14] and ECOG $[10,15]$, the median survival times for patients with SCLC treated with a total radiation dose of $45 \mathrm{~Gy}$ (Table 1), given once or twice daily, approach 2 years, with actuarial 2-year survivals of 40 to $60 \%$.

The current ECOG trial 3588 used only four cycles of cisplatin $60 \mathrm{mg} / \mathrm{m}^{2}$ on day 1 and etoposide $120 \mathrm{mg} / \mathrm{m}^{2}$ on days 1,2 and 3. In contrast, the earlier Penn [5] and ECOG $[10,15]$ trials used five cycles of these drugs alternated with CAV (cyclophosphamide/doxorubicin/vincristine) as maintenance therapy. Prophylactic cranial irradiation (PCI) was provided to complete responders, but was administered at the end of therapy. In ECOG trial 3588, cisplatin/etoposide was given concurrently with thoracic radiotherapy, but patients were randomized to 45 Gy either twice daily for 3 wceks or once daily for 5 weeks (Table 2). Very few differences are worthy of note concerning acute and late effects. Responses were the best seen in any ECOG trial, and myelosuppression, esophageal toxicity, and the incidence of toxic deaths were similar in the two groups.

The effect of hypofractionation was evaluated in a recent study by Prendiville et al. [16], who compared thoracic radiotherapy administered as a single 12.5-Gy dose, with 10 frac- 
PROGRESSION-FREE SURVIVAL: PENN

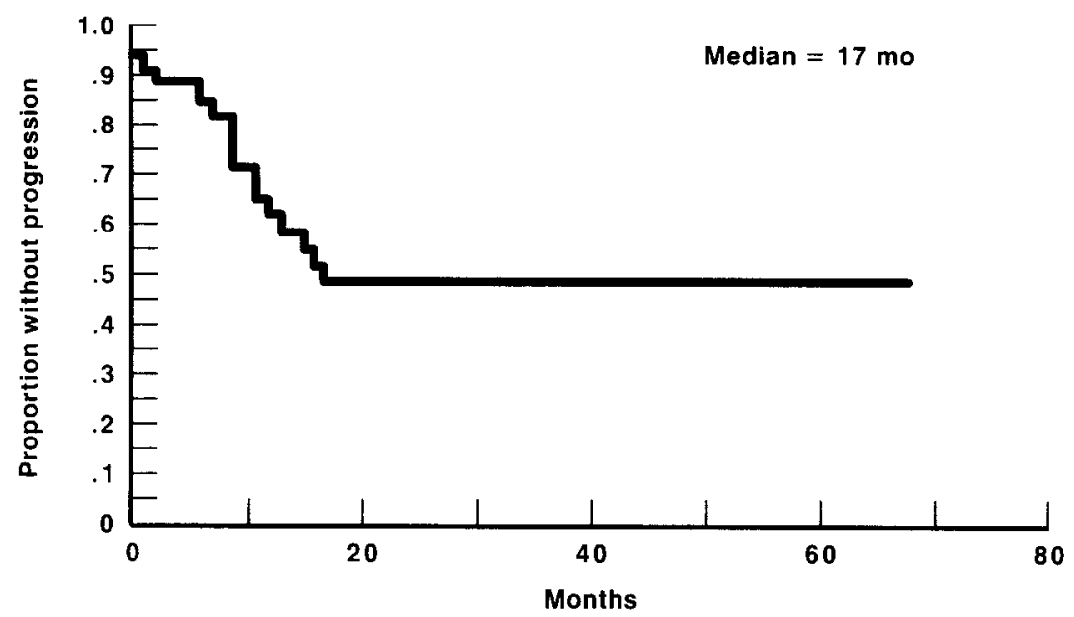

Fig. 3. Progression-free survival in patients receiving twice-daily radiation concurrent with cisplatin/etoposide.

tions of $2.75 \mathrm{~Gy}$, for a $27.5-\mathrm{Gy}$ total dose. Acute toxicity was essentially similar between the two groups, but late toxicity in the group receiving the single dose was almost twice that associated with the hypcrfractionation regimen. Acute toxicity for these infrequent fractionation groups was similar to that reported in twice-daily radiotherapy trials, but these late effects were not seen. In most trials using a total dose of $45 \mathrm{~Gy}$ (given once or twice daily) concurrent with cisplatin/etoposide chemotherapy, the local failure rate is reported to be below $10 \%$. So, isoeffect acute end points with different radiotherapy schedules did not produce similar late effects. Hypofractionated schedules produce poorer local control and excess late effects when compared with standard, accelerated, or hyperfractionated schedules. In the Prendiville trial [16], PCI was optional; approximately $26 \%$ of patients had metastases to the brain, similar to the $20 \%$ actuarial rate associated with use of PCI and substantially less than the $40 \%$ incidence when PCI is omitted.

TABLE 1

Chemotherapy with concurrent thoracic radiotherapy: results of trials in patients with limited SCLC.

\begin{tabular}{lcllll}
\hline Reference & No. patients & Chemotherapy & $\begin{array}{l}\text { Radiation } \\
\text { dose (Gy) }\end{array}$ & & Survival \\
\cline { 5 - 6 } & & & & Median (months) & 2-Year (\%) \\
\hline SWOG [13] & 154 & PEV & 45 daily & 18 & 45 \\
Penn [5] & 32 & PE & 45 b.i.d. & 23 & 45 \\
NCI [14] & 23 & PE & 45 b.i.d. & 24 & 60 \\
ECOG I [10] & 37 & PE & 45 b.i.d. & 22 & 40 \\
ECOG II [15] & 34 & FE & 45 & 24 & 40
\end{tabular}

PEV, cisplatin/etoposide/vincristine; alt, alternating with. 
TABLE 2

Relative scales: once- vs. twice-daily dosing (total dose, $45 \mathrm{~Gy}$ )

\begin{tabular}{llllllll}
\hline Method & No. fractions & $\begin{array}{l}\text { Dose } \\
\text { (Gy) }\end{array}$ & $\begin{array}{l}\text { No. treatment } \\
\text { days }\end{array}$ & RET & Gy-10 & N-RET & Gy-3 \\
\hline Once daily & 25 & 1.8 & 33 & 1415 & 53 & 666 & 72 \\
Twice daily & 30 & 1.5 & 19 & 1438 & 52 & 844 & 68
\end{tabular}

RET, radiation equivalent therapy, a measure of acute effects; NRET, neurolytic RET, a measure of late effects.

\section{Conclusions}

Some therapeutic gains have been offset by excess toxicity. This paper has reviewed the conceptual basis for adding thoracic radiotherapy to systemic chemotherapy to treat limited SCLC. The choice of chemotherapy clearly influences the tolerance. In the 1990s, cisplatin/etoposide regimens can be integrated with radiotherapy with less toxicity and apparently more efficacy.

Methods of administering the thoracic radiotherapy may also influence outcomes. Large volumes and large fraction sizes lead to large frequencies of untoward effects. Trimmed volumes compromise inclusion of lymph nodes but allow for more intense irradiation to areas of bulk that may harbor resistant cells inaccessible or unresponsive to chemotherapy.

PCI provides an opportunity to eliminate those cells that have a relative sanctuary from chemotherapy. It has been used for 20 years, but has a frequency of late effects and fails to influence survival. Nevertheless, it clearly reduces the devastating effects of brain metastasis. Because of this controversy, further studies are needed to settle the debate.

In summary, targeted fractionated thoracic radiotherapy integrated in the first weeks or months of SCLC chemotherapy with cisplatin/etoposide regimens is associated with better survival and local control than have been seen in historical controls. The use of PCI is contentious, and no clear survival advantages are afforded.

\section{References}

1 Bleehen NM, Bunn PA, Cox JD, Dombernowsky P, Fox RM, Host H, Joss R, White JE, Wittes RE. Role of radiation therapy in small-cell anaplastic carcinoma of the lung. Cancer Treat Rep 1983; 67: 11-19.

2 Choi NC, Carey RC. Locoregional failure rate in relation with radiation dose in combined modality approach of multiagent chemotherapy and radiotherapy for limited small-cell lung carcinoma [abstract]. Int J Radiat Oncol Biol Phys 1987; 13 (Suppl) 188.

3 Comis RL. Small-cell carcinoma of the lung. Cancer Treat Rev 1982; 9: 237-258.

4 Carney DN, Mitchell JB, Kinsella TJ. In vitro radiation and chemosensitivity of established cell lines of human small-cell lung cancer and its large cell morphological variants. Cancer Res 1983; 43: 2806-2811.

5 Turrisi AT III, Glover DJ. Thoracic radiotherapy variables: influence on local control in small-cell lung cancer limited disease. Int J Radiat Oncol Biol Phys 1990; 19: 1473-1479.

6 Kies MS, Mira JG, Crowley JJ, Chen TT, Pazdur R, Grozea PN, Rivkin SE, Coltman CA Jr, Ward JH, Livingston RB. Multimodal therapy for limited small-cell lung cancer: a randomized study of induction 
combination chemotherapy with or without thoracic radiation in complete responders; and with wide-field versus reduced-field radiation in partial responders: a Southwest Oncology Group study. J Clin Oncol 1987; 5: $592-600$.

7 Perry MC, Eaton WL, Propert KJ, Ware JH, Zimmer B, Chahinian AP, Skarin A, Carey RW, Kreisman H, Faulkner C, Comis R, Green MR. Chemotherapy with or without radiation therapy in limited small-cell carcinoma of the lung. N Engl J Med 1987; 316: 912-918.

8 Murray N, Coy P, Pater J, Hodson I, Arnold A, Zee B, Payne D, Kostashuk E, Evans W, Dixon P, Sadaura A, Feld R, Lovitt M, Wierzbicki R, Ayoub J, Maroun I, Wilson KS. Importance of timing for thoracic irradiation in combined modality treatment of limited-stage small-cell lung cancer. J Clin Oncol 1993; 11 : 336-344.

9 Catane R, Lichter A, Lee YJ, Brereton HD, Schwade JG, Glatstein E. Small-cell lung cancer: analysis of treatment factors contributing to prolonged survival. Cancer 1981; 48: 1936-1943.

10 Turrisi A, Wagner H, Glover D, Mason B, Oken M, Bonomi P. Limited small-cell lung cancer (LSCLC): concurrent bid thoracic radiotherapy (TRT) with platinum-etoposide (PE): an ECOG study.

11 Johnson BE, Turrisi AT, Chang AY, Blum R, Bonomi P, Ehinger D, Wagner H. Alternating chemotherapy and twice-daily thoracic radiotherapy in limited-stage small-cell lung cancer: a pilot study of the Eastern Cooperative Group. J Clin Oncol 1993; 11: 879-884.

12 Einhorn LH, Crawford J, Birch R, Omura G, Johnson DH, Greco FA. Cisplatin plus etoposide consolidation following cychophosphamide, doxorubicin, and vincristine in limited small-cell lung cancer. J Clin Oncol 1988; 6: 451-456.

13 McCracken JD, Janaki LM, Crowley JJ, Taylor SA, Giri PG, Weiss GB, Gordon W Jr, Baker LH, Mansouri A, Kuebler JP. Concurrent chemotherapy/radiotherapy for limited small-cell lung carcinoma: a Southwest Oncology Group Study. J Clin Oncol 1990; 8: 892-898.

14 Johnson BE, Salem C, Nesbitt J, Gazdar A, Lesar M, Phelps R, Edison M, Linnola R, Phares J, Pass H, Mulshine J, Minna J, Glatstein E, Ihde D. Limited (Ltd) stage small-cell lung cancer (LSCLC) treated with concurrent BID chest radiotherapy (RT) and ctopside cisplatin (VP/PT) followed by chemotherapy (CT) selected by in vitro drug sensitivity testing (DST) [abstract 565]. Lung Cancer 1991; 7 Suppl: 152.

15 Pignon JP, Arriagada R, Ihde D, Johnson DH, Perry MC et al. A metaanalysis of thoracic radiotherapy for small cell lung cancer. N Engl J Med 1992; 327: 1618-1624.

16 Prendiville J, Radford J, Thatcher N, Steward W, Ransom M, Burt P, Stout R. Intensive therapy for smallcell lung cancer using carboplatin alternating with cisplatin, ifosfamide, etoposide, mid-cycle vincristine, and radiotherapy. J Clin Oncol 1991; 9: 1446-1452.

17 Turrisi AT, Glover DJ, Mason B, Tester W. Long term results of platinum etopside (PE) + twice daily (BID) thoracic radiotherapy (TRT) for limited small-cell lung cancer (SCLC): results on 32 patients with 48 month minimum follow-up (FU) [abstract 975]. Proc Am Soc Clin Oncol 1992; 11: 292. 